\title{
Detailed kinetic model describing new oligosaccharides synthesis using different $\beta$-galactosidases
}

\author{
Maria Rodriguez-Fernandez ${ }^{\mathrm{a}, *, 1}$, Alejandra Cardelle-Cobas ${ }^{\mathrm{b}, 1}$, Mar Villamiel $^{\mathrm{c}}$, Julio R. Banga $^{\mathrm{a}}$ \\ a (Bio)Process Engineering Group, Instituto de Investigaciones Marinas (CSIC), Eduardo Cabello, 6, 36208 Vigo, Spain \\ ${ }^{\mathrm{b}}$ Centro de Biotecnologia e Quimica Fina(CBCF)/Escola Superior de Biotecnologia, Universidad Católica Portuguesa, Dr. António Bernardino de Almeida, P-4200-072 Porto, Portugal \\ c Instituto de Investigación en Ciencias de la Alimentación, CSIC-UAM, Nicolás Cabrera, 9, 28049 Madrid, Spain
}

Keywords:

Kinetic models

Lactulose

Transgalactosylation

Model selection

Parameter estimation

Identifiability analysis

\begin{abstract}
A B S T R A C T
The production of prebiotic galactooligosaccharides (GOS) from lactose has been widely studied whereas the synthesis of new prebiotic oligosaccharides with improved properties as those derived from lactulose is receiving an increasing interest. Understanding the mechanism of enzymatic oligosaccharides synthesis from lactulose would help to improve the quality of the products in a rational way as well as to increase the production efficiency by optimally selecting the operating conditions. A detailed kinetic model describing the enzymatic transgalactosylation reaction during lactulose hydrolysis is presented here for the first time. The model was calibrated with the experimental data obtained in batch assays with two different $\beta$-galactosidases at various temperatures and concentrations of substrate. A complete system identification loop, including model selection, robust estimation of the parameters by means of a global optimization method and computation of confidence intervals was performed. The kinetic model showed a good agreement between experimental data and predictions for lactulose conversion and provided important insights into the mechanism of formation of new oligosaccharides with potential prebiotic properties.
\end{abstract}

\section{Introduction}

The increased awareness about the relationship between the activity of colon bacteria and health has lead to the enrichment of some food with prebiotics, defined as "selectively fermented ingredients that allow specific changes, both in the composition and/or activity in the gastrointestinal microflora that confer benefits upon host well-being and health" (Roberfroid, 2007). The beneficial properties of galactooligosaccharides (GOS) on the gut microflora, particularly as prebiotics, are well-known and a number of studies about their enzymatic production by the conversion of lactose catalyzed by $\beta$-galactosidases from different origin have been addressed (Iwasaki et al., 1994; Chen et al., 2003; Kim et al., 2004). The hydrolysis process and the transgalactosylation reaction of lactose take place simultaneously making the mechanism very complicated. Effective models for GOS synthesis are of great interest since they would allow GOS production to be optimized (Gosling et al., 2010), thus, different authors have developed kinetic models

\footnotetext{
* Corresponding author. Current address: Department of Chemical Engineering, University of California Santa Barbara, Santa Barbara, CA 93106-5080, USA. Tel.: +34986231930.

E-mail address: mrodriguez@iim.csic.es (M. Rodriguez-Fernandez).

1 These two authors contributed equally to this work.
}

to explain the formation of these interesting compounds (Mahoney, 1998; Boon et al., 1999; Kim et al., 2004). However, data fitting for this type of models can be very challenging and the identifiability of candidate models should be checked in parallel to model calibration. Further, the standard use of local optimization methods for parameter estimation, such as Levenberg-Marquardt, can result in convergence to local solutions. Therefore, a proper derivation of kinetic models for this type of systems requires the analysis of possible correlations among parameters and the utilization of robust and efficient methods for model calibration.

Prebiotic carbohydrates escape digestion in the upper gastrointestinal tract and are fermented by bacteria in the colon, leading to the proliferation of bacteria that are beneficial for health in humans. Because the place where fermentation mainly occurs (proximal or distal colon) is an important factor influencing the extent of the prebiotic effect (Delzenne, 2003), the development of new types of functional carbohydrates with specific fermentation properties seems to be of interest. Lactulose is a well known disaccharide with excellent prebiotic activity that it is mainly consumed by the bacteria of the proximal colon (Tuohy et al., 2002). Therefore, it is reasonable to assume that lactulose-derived oligosaccharides originated during enzymatic hydrolysis of lactulose might be bioactive carbohydrates slowly fermented and, therefore, with higher colonic persistence than lactulose (CardelleCobas et al., 2008). Thus, recently, in our research group, enzymatic 
transgalactosylation of lactulose with $\beta$-galactosidases from the commercial preparations Lactozym 3000L HP-G and Pectinex Ultra SP-L was studied and new structures such as $6^{\prime}$-galactosyl-lactulose and 1-galactosyl-lactulose were characterized for the first time (Martinez-Villaluenga et al., 2008; Cardelle-Cobas et al., 2008). In spite of these studies, no systematic investigation has been done on the kinetic of formation of these compounds. Therefore, we focused the present study on the development of a mathematical model to describe the kinetics of oligosaccharide synthesis and lactulose hydrolysis with the $\beta$-galactosidases from Lactozym 3000L HP G and Pectinex Ultra SP-L at various temperatures and substrate concentrations.

\section{Materials and methods}

\subsection{Batch reactions}

Time-course reactions for hydrolysis and transgalactosylation of lactulose with the $\beta$-galactosidase from Pectinex Ultra SP-L produced by Aspergillus aculeatus were carried out at $333 \mathrm{~K}$ for $24 \mathrm{~h}$ with initial lactulose concentrations of $0.73,1.33$ and $1.93 \mathrm{M}$, in $0.1 \mathrm{M}$ sodium phosphate buffer, $\mathrm{pH} 6.5$ and $16 \mathrm{U} / \mathrm{mL}$ of $\beta$ galactosidase. The influence of temperature was studied at 313 , 323 and $333 \mathrm{~K}$ with $1.33 \mathrm{M}$ as initial concentration of lactulose (Cardelle-Cobas et al., 2008).

For the $\beta$-galactosidase of Lactozym 3000L HP-G produced by Kluyveromyces lactis, the assays were carried out with an initial concentration of lactulose of $0.73,1.33$ and $1.93 \mathrm{M}$ in $0.05 \mathrm{M}$ potassium phosphate buffer and $1 \mathrm{mM}$ of $\mathrm{MgCl}_{2}, \mathrm{pH} 6.5,3 \mathrm{U} / \mathrm{mL}$ of enzyme, $323 \mathrm{~K}$ and $24 \mathrm{~h}$ of reaction (Martinez-Villaluenga et al., 2008). The influence of temperature was also studied with $0.73 \mathrm{M}$ of initial lactulose at 313 and $323 \mathrm{~K}$.

Lactulose solutions were heated before the enzyme was added and maintained at the required temperature throughout all of the experiments. Reactions were performed in individual Eppendorf tubes incubated in an orbital shaker at $300 \mathrm{rpm}$. Samples were taken at different time intervals and the enzyme was inactivated by heating the sample in water bath at $100^{\circ} \mathrm{C}$ for $5 \mathrm{~min}$. The samples were stored at $-18^{\circ} \mathrm{C}$ for subsequent analysis. All assays were performed in duplicate.

The carbohydrate composition of the reaction mixtures was determined by High Performance Liquid Chromatography with Pulsed Amperometric Detection (HPAEC-PAD) on an ICS2500 Dionex system. Acquisition and processing of data were achieved with Chromeleon software version 6.7 (Dionex Corp., Sunnyvale, CA). Separations were performed at room temperature, following the method of Splechtna et al. (2006). Detection time and voltage parameters were set according to waveform A (Dionex. Technical Note 21).

\subsection{Kinetic modeling and estimation of model parameters}

Given a kinetic model and a set of experimental data, the aim of parameter estimation is to calibrate the model so as to reproduce the experimental data in the best possible way. Calibration of nonlinear models is usually a very challenging task due to nonconvexity that may be overcome by the use of global optimization techniques. However, the key question when trying to identify the parameters of a model is not only whether the model fits the experimental data but also whether the computed parameters are uniquely determined (Schittkowski, 2002, 2007). This question is often neglected leading to models that are able to accurately fit the data but with meaningless parameters due to their huge confidence intervals that are not always computed. In order to develop a proper mechanistic model, a complete system identification loop has to be performed (Ljung, 1999). This includes collecting the experimental data, choosing the model structure(s), defining a quality criterion (cost function), optimizing the parameters with respect to the chosen fitting criterion, evaluating the uncertainty of the estimated parameters and validating the results questioning each of the steps in case the model is proven to be inadequate.

\subsubsection{Model selection}

The model was selected on the basis of the existent literature about the lactose hydrolysis and transgalactosylation. Moreover, the principle of parsimony, stating that "entities must not be multiplied beyond necessity" was also taken into account (Posada and Buckley, 2004). In order to select the most adequate among nested models, the Akaike information criterion (Akaike, 1974), containing a penalty function for increase in the number of parameters, was used in this work. On the other hand, sometimes there is prior knowledge indicating a more complex phenomenological model than statistical criteria allows. Thus, simplifying the model structure may lead to arbitrary values for the phenomenological parameters with unrealistic optimistic evaluation of their uncertainty. In these cases, it is advisable to fix the nonidentifiable parameters to some nominal values coming from the literature or other reliable sources and fit the rest of them.

In conclusion, the discrimination between competing models is a delicate task where a compromise between the goodness of the fit, the mechanistic significance and the quality of the estimated parameters in terms of confidence intervals should be achieved.

\subsubsection{Cost function for model calibration}

Once the characterization of the model has been performed, the identification problem is stated as the optimization of a scalar cost function $J(p)$ with respect to the model parameters, $p$. The cost function is usually a certain weighted distance measure between the experimental values corresponding to the measured variables, represented by the vector $\tilde{y}$, and the predicted values for those variables, represented by the vector $y$. Therefore, the optimal value of $p$ will depend on the cost function chosen. The most widely used cost function (Walter and Pronzato, 1997), the weighted least squares criterion, was considered here:

$J(p)=\sum_{i=1}^{N E} \sum_{j=1}^{N V} \sum_{k=1}^{N M_{i j}} \frac{\left(\tilde{y}_{i j k}-y_{i j k}(p)\right)^{2}}{\sigma_{i j k}^{2}}$

where $\sigma_{i j k}$ corresponds to the standard deviation of the experimental data, $N E$ is the number of experiments, $N V$ the number of variables and $N M_{i j}$ the number of sampling points per variable and experiment. In this way, data with high standard deviation will have less impact on the solution than those determined more accurately. The weighting factors can be chosen iteratively. If the minimization of the initially chosen cost function leads to a model with an unsatisfactory behavior in some region, the weighting factors associated with this zone could be increased in order to improve it (at the cost of deteriorating the fit somewhere else).

In this study, the experimental data were fitted to the proposed model using the SSm GO toolbox, a global optimization metaheuristic based on Scatter Search developed for parameter estimation in nonlinear dynamic biochemical systems (Egea et al., 2007; Rodriguez-Fernandez et al., 2006a).

\subsubsection{Identifiability analysis}

In order to guarantee the quality of the estimated parameters, a practical identifiability analysis should be performed. This study aims to answer the question: given a model structure, would the parameters of the model be uniquely identified from the available (limited and noisy) data? (Jacquez and Greif, 1985; Audoly et al., 
2001). There are mainly two aspects to be checked on a detailed identifiability analysis: sensitivity analysis and parameter correlation.

The sensitivity analysis indicates which parameters are the most important and most likely to affect the predictions of the model. For the sake of simplicity, in this work, we applied a linear sensitivity analysis which consists of calculating a linear approximation of how much a variable changes due to a given change in a parameter. In order to make these measures comparable for parameters and states of different order of magnitude, relative measures were used where the sensitivity function is normalized by the value of the parameter and the state:

$S_{\theta, j}=\frac{p_{\theta}}{y_{j}} \frac{\partial y_{j}}{\partial p_{\theta}}$

For very simple cases, the visual analysis of the relative sensitivity plots can be enough to determine the relative importance of the parameters. However, that becomes intractable when the size of the problem increases and a quantitative justification is needed for establishing a parameter ranking. Brun et al. (2001) recommend the use of the measure $\delta_{\theta}^{m s q r}$ as a ranking criterion in the context of weighted least squares estimation:

$\delta_{\theta}^{m s q r}=\sqrt{\sum_{i=1}^{N E} \sum_{j=1}^{N V} \sum_{k=1}^{N M_{i j}} S_{\theta, i j k}^{2}}$

A large value of the sensitivity index means that a change in the parameter $p_{\theta}$ has an important effect on the model outcome. This makes the parameter $p_{\theta}$ identifiable with the data available if all the other parameters are fixed and the larger the sensitivity the more accurately a single parameter can be identified. Therefore, values of critical parameters can be refined while parameters having a little effect can be simplified or even ignored (Karnavas et al., 1993).

Although necessary, high parameter sensitivity is not enough to ensure the identifiability of the model. In the case of several parameters, the sensitivity functions of the parameters have to be linearly independent. In this study, the degree of linear dependence among the sensitivity functions was measured by means of a correlation analysis based on the Fisher Information Matrix (FIM) as described in Rodriguez-Fernandez et al. (2006b). Correlations among parameters close to +1 or -1 , mean that the parameters are not individually identifiable because a change in one parameter can be compensated by changes in the other parameters. In that case, an infinite number of parameter sets fitting the experimental data with the same accuracy would exist, thus the confidence intervals would be very large. For this reason, the model should be reduced by fixing some of the parameters to their nominal values or by properly grouping some sets.

\subsubsection{Confidence intervals}

After fitting the parameters $p$ to the experimental data, it is important to obtain a measure of the quality of the estimators. In principle, the objective is to obtain the probability distribution of the estimated parameters or an adequate characterization of it, for instance, by computing different percentiles of the distribution. However, in most of the cases this distribution is unknown, therefore, it is necessary to obtain an approximation of it.

A widely used method for describing the confidence intervals of the estimated parameters is the one based on the FIM. Nevertheless, this method presents important disadvantages due to its linear nature; therefore in this work, the bootstrap method (Joshi et al., 2006), which provides a more robust approximation, was used.

\section{Results and discussion}

\subsection{Establishment of the kinetic model}

Since no previous studies exist on the mechanism of formation of oligosaccharides derived from lactulose, we first hypothesized that it is similar to the synthesis of GOS from lactose. Thus, according to the models available in the literature (Boon et al., 1999; Kim et al., 2004), lactulose (Lu) would act as both, substrate and glycosyl acceptor being mainly acceptor to form trisaccharides (Tri) when the concentration is high. Moreover, galactose (Gal) would be bound to the free enzyme to form the galactosyl-enzyme complex (EGal) for further transgalactosylation reactions with galactose or fructose (Fru) as acceptors to produce galactosyl-galactose disaccharides (GalGal) and galactosyl-fructose disaccharides (GalFru). In addition, the synthesis of disaccharides and trisaccharides was assumed to be reversible. Unlike other models, mutarotation of galactose (Bakken et al., 1992) and separate production of tri- and tetrasaccharides (Iwasaki et al., 1994) were not taken into account since this would increase the number of parameters leading to identifiability problems. The formation of monosaccharide-enzyme complexes (EI) avoiding the advance of the transgalactosylation reaction was subject to further investigation.

Moreover, the experimental results obtained with the $\beta$ galactosidase from Pectinex Ultra SP-L, showed that the compound 6 '-galactosyl-lactulose was the main trisaccharide formed, while 6-galactobiose was the disaccharide formed in the highest proportion. Therefore, only the formation of galactosyl-galactose (GalGal) disaccharides was taken into account. In addition, the inhibition assays showed that only galactose caused competitive inhibition on the formation of trisaccharides when the lowest concentration of lactulose was used in the experiments.

On the basis of these theoretical considerations and our experimental data, the following scheme was proposed for the reactions with the $\beta$-galactosidases from Pectinex Ultra SP-L:

$$
\begin{array}{ll}
\mathrm{E}+\mathrm{Lu} \underset{k_{-1}}{\stackrel{k_{1}}{\longrightarrow}} \mathrm{ELu} \stackrel{k_{2}}{\longrightarrow} \mathrm{EGal}+\text { Fru } & \text { Formation of E-Gal complex } \\
\mathrm{EGal}+\mathrm{Lu} \underset{k_{-3}}{\stackrel{k_{3}}{\longrightarrow}} \mathrm{E}+\mathrm{Tri} & \text { Formation of trisaccharides }
\end{array}
$$

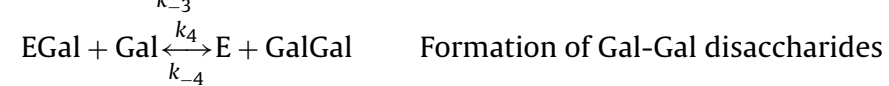

EGal $\underset{k_{-6}}{\stackrel{k_{6}}{\longrightarrow}} \mathrm{E}+\mathrm{Gal} \quad$ Hydrolysis

$\mathrm{E}+\mathrm{Gal} \underset{k_{-7}}{\stackrel{k_{7}}{\longrightarrow}} \mathrm{EI} \quad$ Inhibition by galactose

Once the enzymatic reactions were proposed, and before starting the estimation of parameters, the experimental results were checked based on the sugar residue balance. Thus, it can be determined that, the materials, in each experiment, were conserved for the galactose (Gal) and fructose (Fru) moiety with an error lower than $10 \%$ based on the following conservation equations:

Galactose moiety : $[\mathrm{Lu}]_{0}-[\mathrm{Lu}] \approx[$ Gal $]+2[$ GalGal $]+2[$ Tri $]$ Fructose moiety : $\quad[\mathrm{Lu}]_{0}-[\mathrm{Lu}] \approx[\mathrm{Fru}]+[$ Tri $]$ The balance equation for the enzyme is $[\mathrm{E}]=[\mathrm{E}]_{0}-[\mathrm{EI}]$

$$
-[\text { ELu }]-[\text { EGal }]
$$

where $[\mathrm{Lu}]_{0}$ is the initial mole concentration of substrate.

Thus, the differential equations for the reactions showed above are:

$\frac{d[\mathrm{Lu}]}{d t}=-k_{1}[\mathrm{Lu}][\mathrm{E}]+k_{-1}[\mathrm{ELu}]-k_{3}[\mathrm{EGal}][\mathrm{Lu}]+k_{-3}[\mathrm{Tri}][\mathrm{E}]$ 


$$
\begin{aligned}
\frac{d[\mathrm{Gal}]}{d t}= & -k_{4}[\mathrm{EGal}][\mathrm{Gal}]+k_{-4}[\mathrm{GalGal}][\mathrm{E}]+k_{6}[\mathrm{EGal}]-k_{-6}[\mathrm{Gal}][\mathrm{E}] \\
& -k_{7}[\mathrm{Gal}][\mathrm{E}]+k_{-7}[\mathrm{EI}]
\end{aligned}
$$

$\frac{d[\mathrm{Fru}]}{d t}=k_{2}[\mathrm{ELu}]$

$\frac{d[\mathrm{Tri}]}{d t}=k_{3}[\mathrm{EGal}][\mathrm{Lu}]-k_{-3}[\mathrm{Tri}][\mathrm{E}]$

$\frac{d[\text { GalGal }]}{d t}=k_{4}[$ EGal $][\mathrm{Gal}]-k_{-4}[\mathrm{GalGal}][\mathrm{E}]$

$$
\begin{aligned}
\frac{d[\mathrm{E}]}{d t}= & -k_{1}[\mathrm{Lu}][\mathrm{E}]+k_{-1}[\mathrm{ELu}]+k_{3}[\mathrm{EGal}][\mathrm{Lu}]-k_{-3}[\mathrm{Tri}][\mathrm{E}] \\
& +k_{4}[\mathrm{EGal}][\mathrm{Gal}]-k_{-4}[\mathrm{E}][\mathrm{GalGal}]+k_{6}[\mathrm{EGal}]-k_{-6}[\mathrm{E}][\mathrm{Gal}] \\
& +k_{7}[\mathrm{E}][\mathrm{Gal}]-k_{-7}[\mathrm{EI}]
\end{aligned}
$$

$$
\frac{d[\mathrm{ELu}]}{d t}=k_{1}[\mathrm{Lu}][\mathrm{E}]-k_{-1}[\mathrm{ELu}]-k_{2}[\mathrm{ELu}]
$$

$$
\begin{aligned}
\frac{d[\mathrm{EGal}]}{d t}= & k_{2}[\mathrm{ELu}]-k_{3}[\mathrm{EGal}][\mathrm{Lu}]+k_{-3}[\mathrm{Tri}][\mathrm{E}]-k_{4}[\mathrm{EGal}][\mathrm{Gal}] \\
& +k_{-4}[\mathrm{E}][\mathrm{GalGal}]-k_{6}[\mathrm{EGal}]+k_{-6}[\mathrm{E}][\mathrm{Gal}]
\end{aligned}
$$

$$
\frac{d[\mathrm{EI}]}{d t}=k_{7}[\mathrm{Gal}][\mathrm{E}]-k_{-7}[\mathrm{EI}]
$$

In order to validate the proposed model, Eqs. (4)-(12) were fitted to the available data from the experiments with the $\beta$-galactosidase of Pectinex Ultra SP-L at different lactulose concentrations (0.73, 1.33 and $1.93 \mathrm{M}$ ), temperature being constant (333 K), aiming to find the best set of parameters able to describe the oligosaccharides synthesis at any initial concentration of lactulose among the considered range.

Some of the studies found in the literature (Chen et al., 2003) consider the quasi-steady-state approach for the intermediate complexes (in this work EGal and ELu). Therefore, in this preliminary screening, it was also assumed that $d[$ EGal $] / d t \approx 0$ and $d[\mathrm{ELu}] / d t \approx 0$. The fit obtained following these considerations presented some deficiencies, in particular for the prediction of fructose and galactose concentration so, unlike other authors, the quasisteady-state approximation was not taken into account. In addition, it can be observed that, the enzyme-lactulose (ELu) complex was minimally formed in the reaction mixture in comparison with the EGal complex. Since the ELu almost did not participate in the reaction, it was removed from the model as well as the parameters corresponding to the coefficients for the formation and hydrolysis $\left(k_{1}\right.$ and $\left.k_{-1}\right)$ of ELu.

The relevance of the inhibition by galactose is not clear in the literature. While authors as Mozaffar et al. (1984) and Bakken et al. (1992) claim its importance, others as Boon et al. (1999) consider it negligible under their experimental conditions. In this work, the model without inhibition was considered as a submodel of the one with inhibition and the Akaike's criterion was computed for both models making use of the experimental data. The value of the criterion for the model including inhibition is higher than the one of the model without it, so it can be said that the fit of the data is not significantly better than that obtained with the model without inhibition. Therefore, the inhibition was not considered on the model and the reactions were consequently simplified.

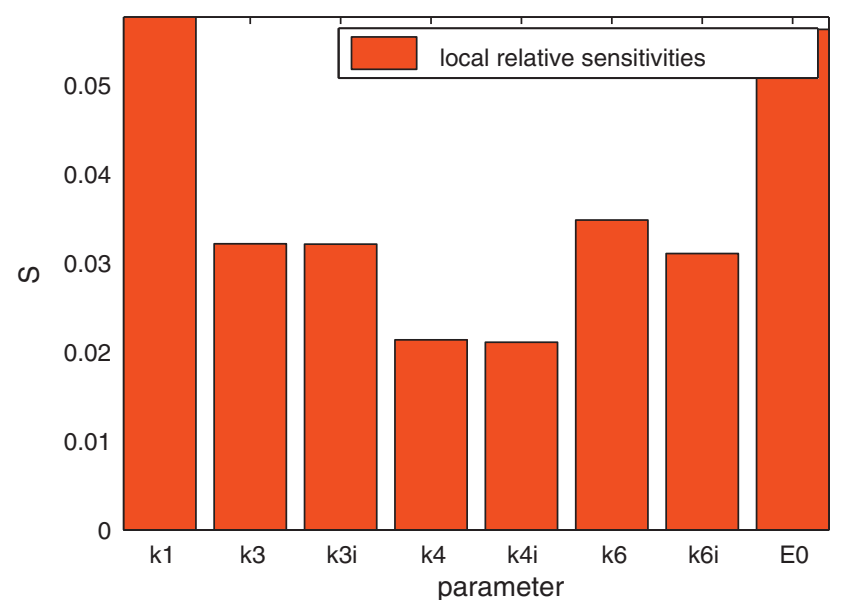

Fig. 1. Local relative sensitivity of the eight parameters involved on the model describing the lactulose conversion with $\beta$-galactosidase of Pectinex Ultra SP-L at constant temperature.

\subsection{Model calibration for the Pectinex experiments}

Once the mechanistic model was selected, the resulting equations were fitted to the data of three experiments with the $\beta$-galactosidase of Pectinex Ultra SP-L at the different lactulose concentrations $(0.73,1.33$ and $1.93 \mathrm{M})$ at $333 \mathrm{~K}$. The measured species were lactulose, fructose, trisaccharides, galactose and galactosylgalactose disaccharides at six sampling times $(0,1,3,5,7$ and $24 \mathrm{~h})$. The kinetic coefficients $\left(k_{1}, k_{3}, k_{-3}, k_{4}, k_{-4}, k_{6}\right.$ and $\left.k_{-6}\right)$ and the initial concentration of the enzyme were optimized by means of the SSm GO toolbox, using the weighted least squares criterion as cost function. The data predicted with the estimated set are in good agreement with the experimental data for the five species measured, so it was used to analyze the practical model identifiability.

The sensitivity analysis (see Fig. 1) shows that the model is influenced by changes on each of the eight estimated parameters. However, the correlation matrix presented in Fig. 2 indicates that the correlations between $k_{3}, k_{4}$ and $k_{6}$ and their reverse coefficients $\left(k_{-3}, k_{-4}\right.$ and $k_{-6}$, respectively) are very high. In order to minimize this identifiability problem, the reverse constants $\left(k_{-3}\right.$, $k_{-4}$ and $k_{-6}$ ) were set to a nominal value and only the direct ones were estimated. As a result, almost the same fit was achieved (only $3 \%$ worse) and the identifiability and confidence intervals

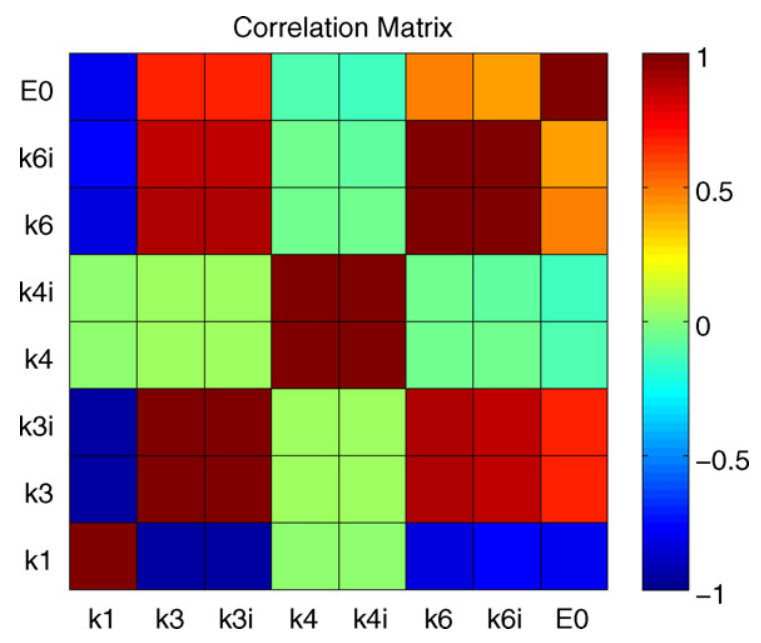

Fig. 2. Correlation matrix of the eight parameters involved on the model describing the lactulose conversion with $\beta$-galactosidase of Pectinex Ultra SP-L at constant temperature. 
Table 1

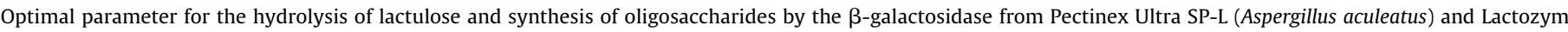
3000L HP G (Kluyveromyces lactis).

\begin{tabular}{|c|c|c|}
\hline \multirow[t]{2}{*}{ Parameter } & Estimated values & Estimated values \\
\hline & A. Aculeatus (Pectinex Ultra SP-L) & K. Lactis (Lactozym 3000L HP G) \\
\hline$k_{1}$ & $3.6 \times 10^{0} \pm 7.8 \times 10^{-1} \mathrm{M}^{-1} \mathrm{~h}^{-1}$ & $2.2 \times 10^{2} \pm 5.9 \times 10^{1} \mathrm{M}^{-1} \mathrm{~h}^{-1}$ \\
\hline$k_{3}$ & $8.8 \times 10^{0} \pm 1.6 \times 10^{0} \mathrm{M}^{-1} \mathrm{~h}^{-1}$ & $3.4 \times 10^{-1} \pm 8.4 \times 10^{-2} \mathrm{M}^{-1} \mathrm{~h}^{-1}$ \\
\hline$k_{-3}$ & $1.0 \times 10^{2} \mathrm{M}^{-1} \mathrm{~h}^{-1}$ & $1.0 \times 10^{2} \mathrm{M}^{-1} \mathrm{~h}^{-1}$ \\
\hline$k_{4}$ & $1.8 \times 10^{0} \pm 3.4 \times 10^{-1} \mathrm{M}^{-1} \mathrm{~h}^{-1}$ & $2.9 \times 10^{-1} \pm 7.7 \times 10^{-2} \mathrm{M}^{-1} \mathrm{~h}^{-1}$ \\
\hline$k_{-4}$ & $1.0 \times 10^{2} \mathrm{M}^{-1} \mathrm{~h}^{-1}$ & $1.0 \times 10^{2} \mathrm{M}^{-1} \mathrm{~h}^{-1}$ \\
\hline$k_{6}$ & $3.3 \times 10^{0} \pm 6.3 \times 10^{-1} \mathrm{M}^{-1} \mathrm{~h}^{-1}$ & $4.4 \times 10^{-1} \pm 1.0 \times 10^{-1} \mathrm{M}^{-1} \mathrm{~h}^{-1}$ \\
\hline$k_{-6}$ & $1.0 \times 10^{2} \mathrm{M}^{-1} \mathrm{~h}^{-1}$ & $1.0 \times 10^{2} \mathrm{M}^{-1} \mathrm{~h}^{-1}$ \\
\hline$E_{0}$ & $1.5 \times 10^{-1} \pm 1.5 \times 10^{-2} \mathrm{M}$ & $1.0 \times 10^{-1} \pm 1.9 \times 10^{-2} \mathrm{M}$ \\
\hline
\end{tabular}

were strongly improved. Since no relevant values for the inverse constants were found in the literature, the assigned ones were somehow arbitrary. The value of the estimated constants and their confidence intervals for the best fit are shown in Table 1. As can be observed in Fig. 3, the fits for this enzyme for different initial concentrations of lactulose $(0.73 \mathrm{M}(\mathrm{A}), 1.33 \mathrm{M}(\mathrm{B})$ and $1.93 \mathrm{M}(\mathrm{C})$ ), showed a good agreement between the predicted and the experimental data. Moreover, the mathematical model is able to accurately predict the higher production of disaccharides and trisaccharides when the initial concentration of lactulose increases.

\subsection{Model calibration for the Lactozym experiments}

Since the developed model was able to fit the experimental data corresponding to the synthesis of lactulose-derived oligosaccharides with the $\beta$-galactosidase from Pectinex Ultra SP-L, it was interesting to analyze if the same model could describe the synthesis of oligosaccharides with the $\beta$-galactosidase of Lactozym 3000L HP G used in previous studies about the formation of new compounds using lactulose as substrate (Martinez-Villaluenga et al., 2008). For this enzyme, experimental data at different concentrations of lactulose (0.73-1.93 M) and different temperatures (313 and $323 \mathrm{~K}$ ) were also available. The chromatographic profile obtained with this enzyme was quite similar to that obtained for the synthesis of oligosaccharides with Pectinex Ultra SP-L but the amount of compounds was different. Thus, in these assays, two trisaccharides were obtained in a major proportion, the $6^{\prime}$ galactosyl-lactulose and the 1-galactosyl-lactulose. In this way, the same kinetic model with the same assumptions (absence of inhibition at high concentrations of Lu, formation of galactosyl-galactose type disaccharides, etc.) was fitted to the data corresponding to three experiments at the same temperature (323 K) and different initial concentrations of lactulose $(0.73,1.33$ and $1.93 \mathrm{M})$.

The production of disaccharides was small and it increased with increasing time of reaction for both enzymes at all temperatures. This production showed a pretty linear trend for Pectinex Ultra SP-L but for Lactozym 3000L HP G the concentration seemed to be zero or at least non-measurable during the first 4-6h at high concentrations of enzyme, presenting sudden increases afterwards. This made the fitting of the disaccharides very difficult with the available model and, at the same time, they strongly influence the value of the objective function due to the small standard deviation of these points (despite the fact that at this low concentration the experimental errors are known to be quite high). That makes the estimation to neglect other species that are more important for this study, so the weights of the objective function were manually tuned in order to avoid this bias.

The sensitivity analysis for the best set of parameters obtained with these data and the tuned objective function showed a significantly smaller sensitivity of the model output to the reverse parameters $\left(k_{-3}, k_{-4}\right.$ and $\left.k_{-6}\right)$ than to the direct ones and a very high sensitivity to the initial concentration of the enzyme.
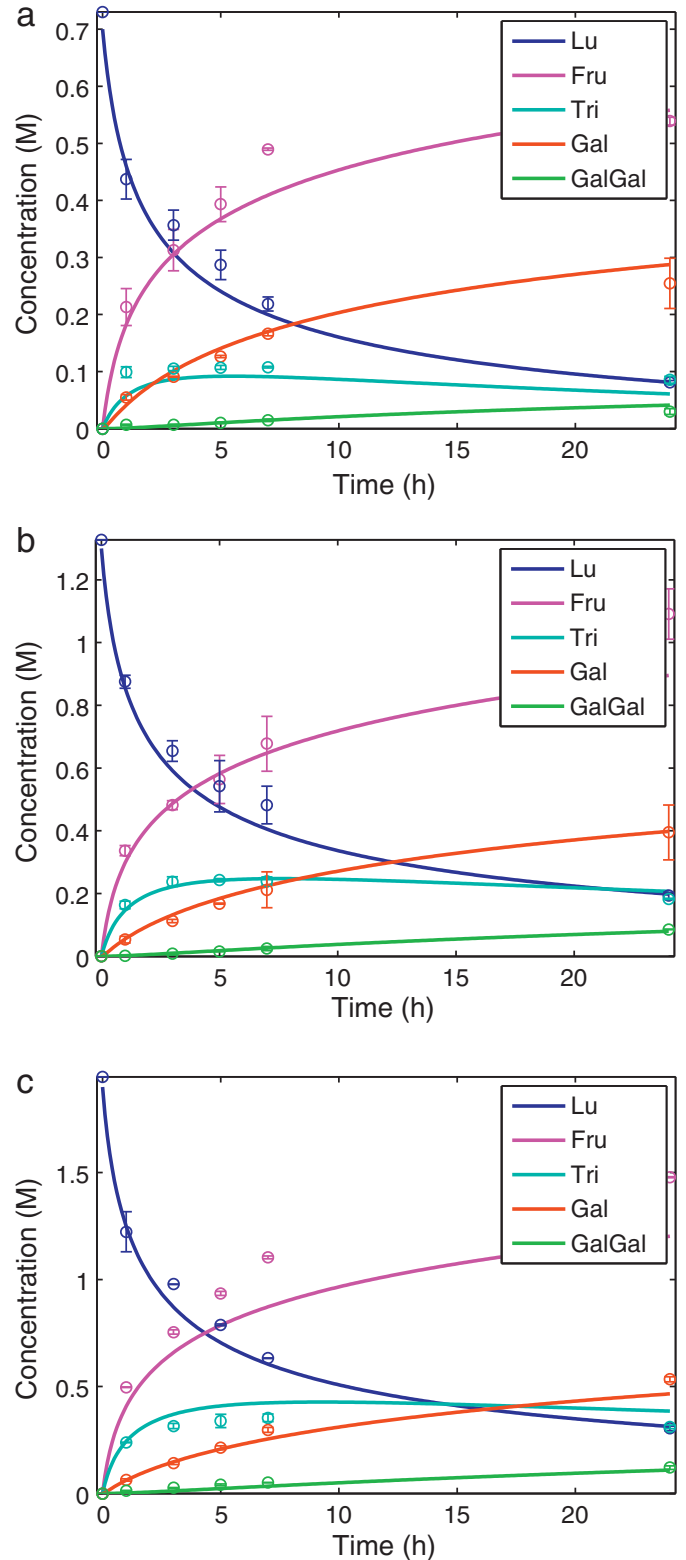

Fig. 3. Time-course reactions for lactulose conversion with $\beta$-galactosidase of Pectinex Ultra SP-L with three different initial concentrations of lactulose (A) $0.73 \mathrm{M}$, (B) $1.33 \mathrm{M}$ and (C) $1.93 \mathrm{M}$ and temperature of $333 \mathrm{~K}$. Symbols are the points of experimental data and lines are the predicted values for the best fit of the quasi-steady state model. 
Moreover the correlation between $k_{1}$ and the reverse constants is equal to one so, following the previous scheme, the reverse constants $\left(k_{-3}, k_{-4}\right.$ and $\left.k_{-6}\right)$ were set to a nominal value.

The value of the best set of parameters and their confidence intervals are shown in Table 1 . As expected, the value found for $k_{1}$ is higher than that estimated for Pectinex Ultra SP-L at $333 \mathrm{~K}$ since the decrease in the concentration of lactulose and the increase in the concentration of fructose are faster for Lactozym 3000L HP G at $323 \mathrm{~K}$. The values found for the formation of trisaccharides $\left(k_{3}\right)$ and disaccharides $\left(k_{4}\right)$ were in the same order of magnitude, which means that, in this case, both galactose and lactulose are good glycosyl acceptors to form these compounds. In contrast, for Pectinex Ultra SP-L at $333 \mathrm{~K}$, the value of $k_{3}$ was higher than $k_{4}$ indicating that lactulose acts as a better acceptor than galactose, which explains the fact that, under the assayed conditions, more trisaccharides are formed at a faster reaction rate. Fig. 4 shows the good agreement found between the experimental data and the predicted values for the best fit, supporting the goodness of the model.

\subsection{Modeling the temperature dependence}

To complete this study, a more complex scheme allowing handling experiments at different temperatures was included into the model. As a first approach, the rate constants $\left(k_{1}, k_{3}, k_{-3}, k_{4}, k_{-4}, k_{6}\right.$ and $k_{-6}$ ) were assumed to follow the Arrhenius equation:

$k=K_{0} e^{-E_{\mathrm{a}} / R T}$

where $E_{\mathrm{a}}$ is the activation energy $(\mathrm{J} / \mathrm{mol}), K_{0}$ the pre-exponential factor, $R$ the ideal gas constant $(8.3 \mathrm{~J} / \mathrm{mol} \mathrm{K})$ and $T$ is the temperature (K). The expression of the Arrhenius equation for the direct and inverse reactions was employed in the model as in the following example:

Formation of trisaccharides : $\quad k_{3}=K_{03} e^{-E_{\mathrm{a} 3} / R T}$

Hydrolysis of trisaccharides : $\quad k_{-3}=K_{03 i} e^{-E_{\mathrm{a} 3 \mathrm{i}} / R T}$

The rate expressions for the proposed model, above mentioned, were fitted simultaneously for the five different experiments carried out with the $\beta$-galactosidase of Pectinex Ultra SP-L at various initial concentrations of substrate $(0.73,1.33$ and $1.93 \mathrm{M})$ and temperatures (313, 323 and $333 \mathrm{~K})$. The concentration of enzyme and the $\mathrm{pH}$ remained constant in all the experiments. Each experiment described the lactulose, fructose, galactose, trisaccharide and disaccharide evolution in time. Fig. 5 shows the experimental data and the model prediction for the three batch experiments. As can be seen, a good agreement was obtained between the experimental and simulated values.

The final concentration of trisaccharides increases as the temperature increases being maximum at $333 \mathrm{~K}$. This phenomenon is captured by the model and reflected on the value of the parameters, $E_{\mathrm{a} 3}$ is greater than $E_{\mathrm{a} 3 \mathrm{i}}$, meaning that the formation of trisaccharides is favored by the temperature. In the case of the disaccharides, the opposite effect is observed; the final concentration is smaller for higher temperatures. Accordingly, $E_{\mathrm{a} 4 \mathrm{i}}$ is larger than $E_{\mathrm{a} 4}$ meaning that the inverse reaction is favored by the temperature more than it is the direct reaction. Therefore, the production of disaccharides decreases at high temperatures.

The same procedure was followed for the synthesis of oligosaccharides by the $\beta$-galactosidase of Lactozym 3000L HP G. Thus, the data coming from four experiments at different lactulose concentrations $(0.73,1.33$ and $1.93 \mathrm{M}$ ) and temperatures ( 313 and $323 \mathrm{~K}$ ) were fitted using the same kinetic model. Simulated values were plotted as continuous lines in Fig. 6, while experimental results are given as symbols, showing the good agreement obtained for this enzyme. In this case the available temperatures were only two,
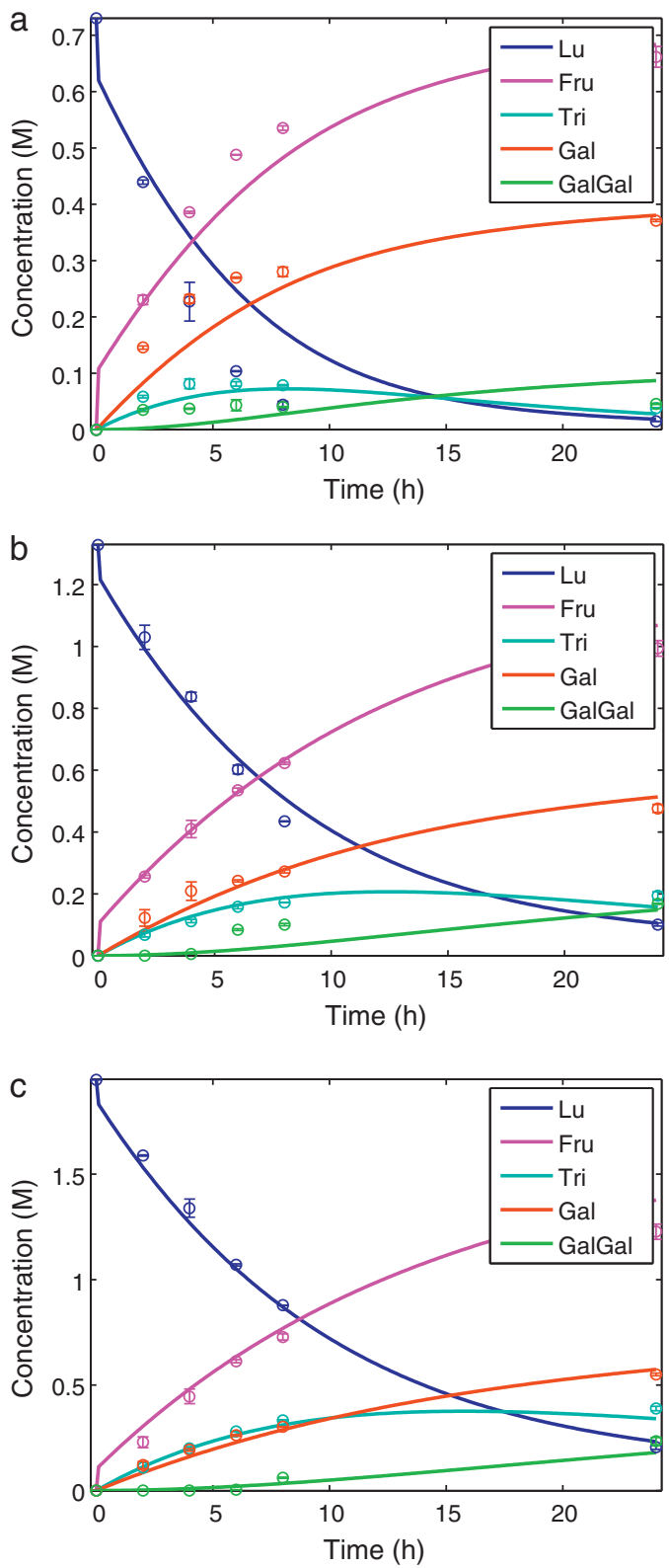

Fig. 4. Time-course reactions for lactulose conversion with $\beta$-galactosidase of Lactozym 3000L HP G with three different initial concentrations of lactulose (A) $0.73 \mathrm{M}$, (B) $1.33 \mathrm{M}$ and (C) $1.93 \mathrm{M}$ and temperature of $323 \mathrm{~K}$.

therefore, the fitting of the model was more challenging and the decreasing of disaccharides formation with the temperature was not captured properly. That explains why $E_{\mathrm{a} 4}$ is larger than $E_{\mathrm{a} 4 \mathrm{i}}$ even if the final concentration of disaccharides is slightly smaller at $323 \mathrm{~K}$ than at $313 \mathrm{~K}$. Regarding the trisaccharides, their behavior is properly captured. The data show that lactulose is a better acceptor of glycosyl at $313 \mathrm{~K}$ than at $323 \mathrm{~K}$ and the estimated parameters point out in the same direction, $E_{\mathrm{a} 3 \mathrm{i}}$ is larger than $E_{\mathrm{a} 3}$. Since the constant of the inverse reaction increases with the temperature, the amount of trisaccharides will decrease.

The numeric values obtained for the best fit in the temperaturedependent model for the two enzymes used in this work are shown in Table 2. It is worth noting that, the correlation found between the different parameters, especially between the pre-exponential factors and their corresponding activation energy for the Arrhenius equations are very close to 1 , so it cannot be stated that these sets 

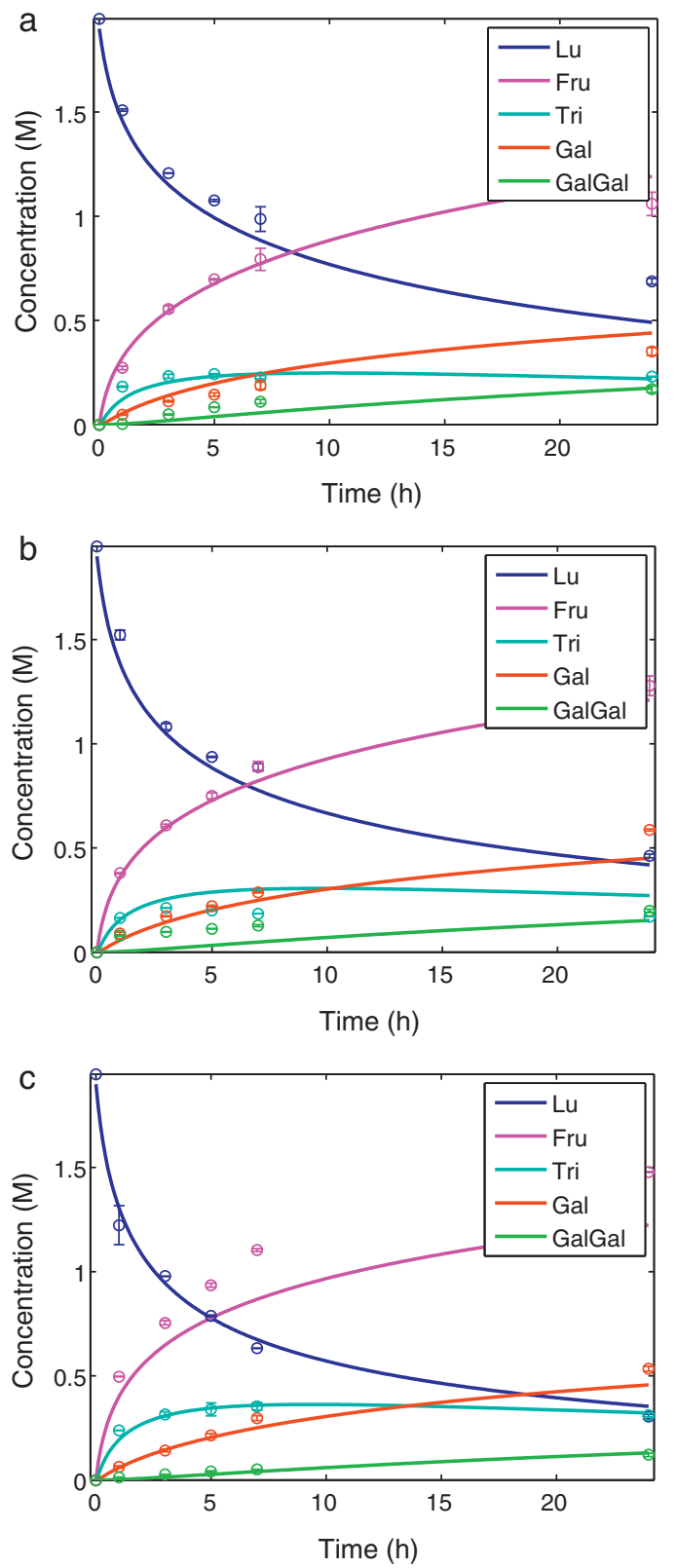

Fig. 5. Time-course reactions for lactulose conversion with $\beta$-galactosidase of Pectinex Ultra SP-L with initial concentration of lactulose of $1.93 \mathrm{M}$ and different temperatures (A) $313 \mathrm{~K}$, (B) $323 \mathrm{~K}$ and (C) $333 \mathrm{~K}$.

of parameters are the only ones providing a good fit to the experimental data. This is due to the difficulties inherent to the Arrhenius equation structure already reported by other authors as Pritchard and Bacon (1978). Moreover, the limited amount of experimental data (i.e. for the $\beta$-galactosidase only data at two different temperatures and with a difference of only $10^{\circ} \mathrm{C}$ ) made the identification even harder.

Table 3 shows the reaction rate coefficients calculated from the best parameters obtained for the proposed model at a fixed temperature. The temperatures selected were those for which a maximum formation of trisaccharides was obtained, i.e., $333 \mathrm{~K}$ for the Pectinex Ultra SP-L and $313 \mathrm{~K}$ for the Lactozym 3000L HP G. Although identifiability results indicate a strong dependence of the direct and reverse constants, we can indicate for all calculated parameters at the most favorable temperature among the range of this study for the formation of trisaccharides, a set of parameters that give an
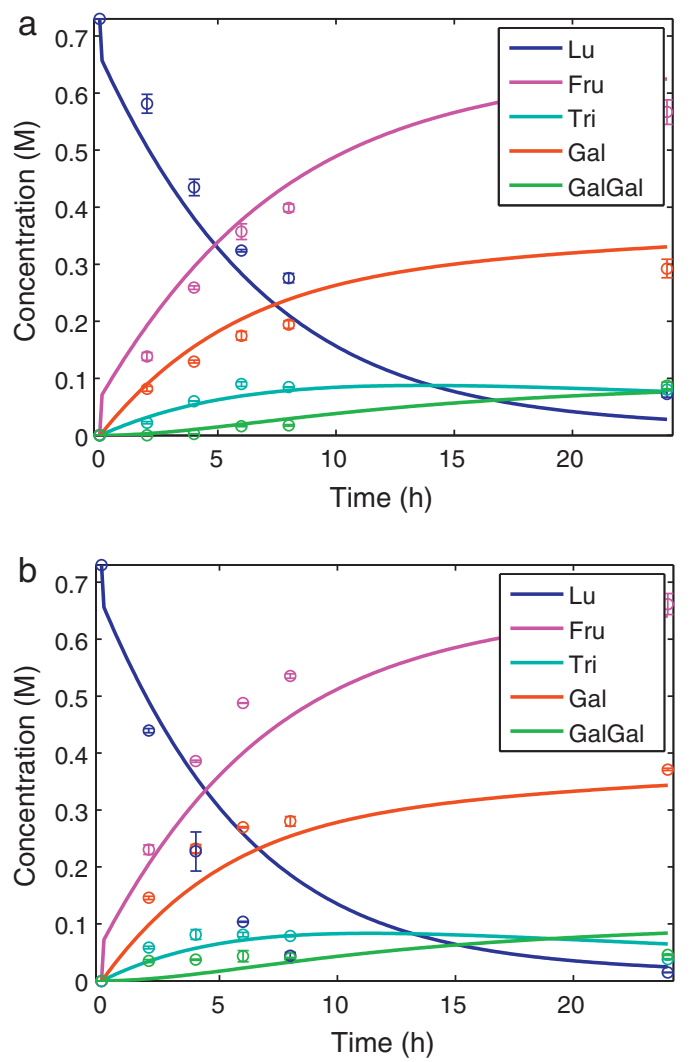

Fig. 6. Time-course reactions for lactulose conversion with $\beta$-galactosidase of Lactozym 3000L HP G with initial concentration of lactulose of $0.73 \mathrm{M}$ and different temperatures (A) $313 \mathrm{~K}$ and (B) $323 \mathrm{~K}$.

approximation of the behavior of both enzymes in the formation of oligosaccharides derived from lactulose. As can be seen in the table, for the $\beta$-galactosidase of Lactozym 3000L HP G the fitted reaction rate coefficient for the formation of the EGal complex was much higher than that obtained for the $\beta$-galactosidase of Pectinex Ultra SP-L, indicating a higher rate of formation and, therefore, a faster decrease in lactulose concentration in the reaction medium when Lactozym 3000L HP G is used as source of $\beta$-galactosidase. For Pectinex Ultra SP-L, the fitted reaction rate coefficient for the

Table 2

Optimal parameter for the hydrolysis of lactulose and synthesis of oligosaccharides by the $\beta$-galactosidase from Pectinex Ultra SP-L (Aspergillus aculeatus) and Lactozym 3000L HP G (Kluyveromyces lactis).

\begin{tabular}{lll}
\hline Parameter & Estimated value & Estimated value \\
\cline { 2 - 3 } & A. aculeatus (Pectinex Ultra SP-L) & $\begin{array}{l}\text { K. lactis (Lactozym } \\
\text { 3000L HP G) }\end{array}$ \\
\hline$k_{01}$ & $3.8 \times 10^{2} \mathrm{M}^{-1} \mathrm{~h}^{-1}$ & $4.8 \times 10^{3} \mathrm{M}^{-1} \mathrm{~h}^{-1}$ \\
$k_{03}$ & $1.9 \times 10^{4} \mathrm{M}^{-1} \mathrm{~h}^{-1}$ & $1.4 \times 10^{1} \mathrm{M}^{-1} \mathrm{~h}^{-1}$ \\
$k_{-03 \mathrm{i}}$ & $1.5 \times 10^{2} \mathrm{M}^{-1} \mathrm{~h}^{-1}$ & $1.1 \times 10^{6} \mathrm{M}^{-1} \mathrm{~h}^{-1}$ \\
$k_{04}$ & $3.2 \times 10^{0} \mathrm{M}^{-1} \mathrm{~h}^{-1}$ & $2.6 \times 10^{0} \mathrm{M}^{-1} \mathrm{~h}^{-1}$ \\
$k_{-04}$ & $5.3 \times 10^{5} \mathrm{M}^{-1} \mathrm{~h}^{-1}$ & $1.2 \times 10^{2} \mathrm{M}^{-1} \mathrm{~h}^{-1}$ \\
$k_{06}$ & $1.9 \times 10^{2} \mathrm{M}^{-1} \mathrm{~h}^{-1}$ & $2.1 \times 10^{1} \mathrm{M}^{-1} \mathrm{~h}^{-1}$ \\
$k_{-06}$ & $1.1 \times 10^{5} \mathrm{M}^{-1} \mathrm{~h}^{-1}$ & $1.4 \times 10^{3} \mathrm{M}^{-1} \mathrm{~h}^{-1}$ \\
$E_{\mathrm{a} 1}$ & $1.3 \times 10^{4} \mathrm{~J} / \mathrm{M}$ & $9.7 \times 10^{3} \mathrm{~J} / \mathrm{M}$ \\
$E_{\mathrm{a} 3}$ & $2.2 \times 10^{4} \mathrm{~J} / \mathrm{M}$ & $9.0 \times 10^{3} \mathrm{~J} / \mathrm{M}$ \\
$E_{\mathrm{a} 3 \mathrm{i}}$ & $1.0 \times 10^{3} \mathrm{~J} / \mathrm{M}$ & $2.8 \times 10^{4} \mathrm{~J} / \mathrm{M}$ \\
$E_{\mathrm{a} 4}$ & $1.0 \times 10^{3} \mathrm{~J} / \mathrm{M}$ & $4.1 \times 10^{3} \mathrm{~J} / \mathrm{M}$ \\
$E_{\mathrm{a} 4 \mathrm{i}}$ & $2.3 \times 10^{4} \mathrm{~J} / \mathrm{M}$ & $3.0 \times 10^{2} \mathrm{~J} / \mathrm{M}$ \\
$E_{\mathrm{a} 6}$ & $1.4 \times 10^{4} \mathrm{~J} / \mathrm{M}$ & $8.4 \times 10^{3} \mathrm{~J} / \mathrm{M}$ \\
$E_{\mathrm{a} 6 \mathrm{i}}$ & $2.2 \times 10^{4} \mathrm{~J} / \mathrm{M}$ & $6.4 \times 10^{3} \mathrm{~J} / \mathrm{M}$ \\
$E_{0}$ & $2.0 \times 10^{-1} \mathrm{M}$ & $6.6 \times 10^{-2} \mathrm{M}$ \\
\hline
\end{tabular}


Table 3

Reaction rate coefficients for the optimal temperature of formation of trisaccharides by the $\beta$-galactosidases from Pectinex Ultra SP-L (333K) and Lactozym 3000L HP G (313 K).

\begin{tabular}{lll}
\hline Reaction rate constants & \multicolumn{2}{l}{ Rate coefficients values } \\
\cline { 2 - 3 } & $\begin{array}{ll}\text { A. aculeatus (Pectinex } \\
\text { Ultra SP-L) }\end{array}$ & $\begin{array}{l}\text { K. lactis (Lactozym } \\
3000 \mathrm{~L} \mathrm{HP}-\mathrm{G} \text { ) }\end{array}$ \\
\hline$k_{1}$ & $2.8 \times 10^{0} \mathrm{M}^{-1} \mathrm{~h}^{-1}$ & $1.1 \times 10^{2} \mathrm{M}^{-1} \mathrm{~h}^{-1}$ \\
$k_{3}$ & $6.4 \times 10^{0} \mathrm{M}^{-1} \mathrm{~h}^{-1}$ & $4.4 \times 10^{-1} \mathrm{M}^{-1} \mathrm{~h}^{-1}$ \\
$k_{-3}$ & $1.1 \times 10^{2} \mathrm{M}^{-1} \mathrm{~h}^{-1}$ & $2.3 \times 10^{1} \mathrm{M}^{-1} \mathrm{~h}^{-1}$ \\
$k_{4}$ & $2.3 \times 10^{0} \mathrm{M}^{-1} \mathrm{~h}^{-1}$ & $5.4 \times 10^{-1} \mathrm{M}^{-1} \mathrm{~h}^{-1}$ \\
$k_{-4}$ & $1.3 \times 10^{2} \mathrm{M}^{-1} \mathrm{~h}^{-1}$ & $1.1 \times 10^{2} \mathrm{M}^{-1} \mathrm{~h}^{-1}$ \\
$k_{6}$ & $1.3 \times 10^{0} \mathrm{M}^{-1} \mathrm{~h}^{-1}$ & $8.3 \times 10^{-1} \mathrm{M}^{-1} \mathrm{~h}^{-1}$ \\
$k_{-6}$ & $4.0 \times 10^{1} \mathrm{M}^{-1} \mathrm{~h}^{-1}$ & $1.2 \times 10^{2} \mathrm{M}^{-1} \mathrm{~h}^{-1}$ \\
\hline
\end{tabular}

formation of trisaccharides $\left(k_{3}\right)$ was found to be higher than that for disaccharides $\left(k_{4}\right)$ and the values found for the reverse reactions $\left(k_{-3}\right.$ and $\left.k_{-4}\right)$ were similar. Therefore, for the $\beta$-galactosidase of Pectinex Ultra SP-L at $333 \mathrm{~K}$, it is possible to establish that lactulose acts as a better glycosyl acceptor to form trisaccharides but these are more easily broken down; galactose is a poorer acceptor than lactulose but has more chances to form disaccharides at lower concentrations of lactulose and the disaccharides originated are much more stable. On the contrary, in the case of Lactozym 3000L HP G, the fitted reaction rate coefficient for trisaccharide formation $\left(k_{3}\right)$ was similar to that found for disaccharides $\left(k_{4}\right)$ being the values obtained for the reverse reactions $\left(k_{-3}, k_{-4}\right)$ strikingly different. Thus, for the $\beta$-galactosidase of Lactozym 3000L HP G, both lactulose and galactose are good glycosyl acceptors to form trisaccharides and disaccharides, being the latter more rapidly hydrolyzed and, therefore, less stable.

\section{Conclusions}

The proposed model can describe the oligosaccharide synthesis using the $\beta$-galactosidases from Pectinex Ultra SP-L (A. aculeatus) and Lactozym 3000L HP-G (K. lactis) at several temperatures and initial concentrations of lactulose. The experimental data are in good agreement with the predictions of the developed model. In accordance with the experimental data, the kinetic parameters describing the reversible oligosaccharide synthesis are of different magnitude for both $\beta$-galactosidases, since they produce different amounts and types of oligosaccharides. The formation of trisaccharide was favored in the synthesis of oligosaccharides using lactulose as substrate and Pectinex Ultra SP-L, whereas the formation of disaccharides was higher when the $\beta$-galactosidase from Lactozym 3000 L HP G was used as enzyme. Moreover, the formation of trisaccharides is larger at high temperatures when using Pectinex Ultra SP-L (maximum at $333 \mathrm{~K}$ ) even though the formation of disaccharides is larger at lower temperatures. In the case of the Lactozym $3000 \mathrm{~L} \mathrm{HP}-\mathrm{G}$, the production of both di- and trisaccharides is higher at $313 \mathrm{~K}$ (the minimum temperature used for the experiments). This is the first time that a complete system identification loop, including model selection using the Akaike criterion, robust estimation of the parameters by means of a global optimization method and computation of confidence intervals is performed for the kinetic study on the formation of new oligosaccharides with potential prebiotic properties. Important insights into the mechanism of formation of new oligosaccharides with potential prebiotic properties were obtained from the developed model that could ultimately be used to select the optimal operating conditions for increasing the efficiency of the production or for the selective formation of a target di- or trisaccharide.

\section{Acknowledgments}

This work was financed under the projects CONSOLIDER INGENIO 2010 FUNC-C-FOOD CSD2007-00063 from MICINN. A. Cardelle-Cobas thanks MEC for FPU grant. Authors M. RodriguezFernandez and J.R. Banga acknowledge financial support from the EU ERASysBio and the Spanish Ministry of Science and Innovation (SYSMO project KOSMOBAC, ref. MEC GEN2006-27747-E/SYS and MICINN project MultiSysBio ref. DPI2008-06880-C03-02).

\section{References}

Akaike, H., 1974. A new look at the statistical model identification. IEEE Transactions on Automatic Control 19 (6), 716-723.

Audoly, S., Bellu, G., D’Angio, L., Saccomani, M.P., Cobelli, C., 2001. Global identifiability of nonlinear models of biological systems. IEEE Transactions on Biomedical Engineering 48 (1), 55-65.

Bakken, A.P., Hill Jr., C.G., Amudson, C.H., 1992. Hydrolysis of lactose in skim milk by immobilized $\beta$-galactosidase (Bacillus circulans). Biotechnology and Bioengineering 39, 408-417.

Boon, M.A., Janseen, A.E.M., van der Padt, A., 1999. Modelling and parameter estimation of the enzymatic synthesis of oligosaccharide synthesis of oligosaccharides by $\beta$-galactosidase from Bacillus circulans. Biotechnology and Bioengineering 64 (5), 558-567.

Brun, R., Reichert, P., Kunsch, H.R., 2001. Practical identifiability analysis of large environmental simulation models. Water Resources Research 37 (4), 1015-1030.

Cardelle-Cobas, A., Martinez-Villaluenga, C., Villamiel, M., Olano, A., Corzo, N., 2008. Synthesis of oligosaccharides from lactulose and Pectinex Ultra SP-L. Journal of Agricultural and Food Chemistry 56, 3328-3333.

Chen, C.W., Ouh-Yang, C.-C., Yeh, C.-W., 2003. Synthesis of galactooligosacharides and transgalactosylation modeling in reverse micelles. Enzyme and Microbial Technology 33, 497-507.

Delzenne, N.M., 2003. Oligosaccharides: state of the art. Proceedings of the Nutrition Society $62,177-182$.

Dionex. Technical Note 21. Optimal settings for pulsed amperometric detection of carbohydrates using the Diones ED40 electrochemical detector.

Egea, J.A., Rodriguez-Fernandez, M., Banga, J.R., Marti, R., 2007. Scatter search for chemical and bioprocess optimization. Journal of Global Optimization 37, 481-503.

Gosling, A., Stevens, G.W., Barber, A.R., Kentish, S.E., Gras, S.L., 2010. Recent advances refining galactooligosaccharide production from lactose. Food Chemistry 121 307-318.

Iwasaki, K., Nakajima, M., Nakao, S., 1994. Galacto-oligosaccharide production from lactose by an enzymic batch reaction using $\beta$-galactosidase. Process in Biochemistry $31,69-76$.

Jacquez, J.A., Greif, P., 1985. Numerical parameter identifiability and estimability: integrating identifiability, estimability, and optimal sampling desing. Mathematical Biosciences 77, 201-227.

Joshi, M., Seidel-Morgenstern, A., Kremling, A., 2006. Exploiting the bootstrap method for quantifying parameter confidence intervals in dynamical systems. Metabolic Engineering 8, 447-455

Karnavas, W.J., Sanchez, P., Bahill, A.T., 1993. Sensitivity analyses of continuous and discrete systems in the time and frequency domains. IEEE Transactions on Systems, Man and Cybernetics 23, 488-501.

Kim, C.S., Ji, E.S., Oh, D.K., 2004. A new kinetic model of recombinant b-galactosidase from Kluyveromyces lactis for both hydrolysis and transgalactosylation reactions. Biochemical and Biophysical Communications 316, 738-743.

Ljung, L., 1999. System Identification: Theory for the User. Prentice Hall.

Mahoney, R.R., 1998. Galactosyl-oligosaccharide formation during lactose hydrolysis: a review. Food Chemistry 63 (2), 147-154.

Martinez-Villaluenga, C., Cardelle-Cobas, A., Olano, A., Corzo, N., Villamiel, M., Jimeno, M.L., 2008. Enzymatic synthesis and identification of two trisaccharides produced from lactulose by transgalactosylation. Journal of Agricultural and Food Chemistry 56, 557-563.

Mozaffar, Z., Nakanishi, K., Matsuno, R., Kamikubo, T., 1984. Purification and properties of b-galactosidases from Bacillus circulans. Agricultural and Biological Chemistry 48, 3053-3061.

Posada, D., Buckley, T.R., 2004. Model selection and model averaging in phylogenetics: advantages of Akaike information criterion and Bayesian approaches over likelihood ratio tests. Systematic Biology 53 (5), 793-808.

Pritchard, D.J., Bacon, D.W., 1978. Prospects for reducing correlations among parameter estimates in kinetic models. Chemical Engineering Science 33, 1539-1543.

Roberfroid, M., 2007. Prebiotics: the concept revisited. Journal of Nutrition 137 (3), 830s-837s.

Rodriguez-Fernandez, M., Egea, J.A., Banga, J.R., 2006a. Novel metaheuristic for parameter estimation in nonlinear dynamic biological systems. BCM Bioinformatics 7, 483

Rodriguez-Fernandez, M., Mendes, P., Banga, J.R., 2006b. A hybrid approach for efficient and robust parameter estimation in biochemical pathways. BioSystems 83 , $248-265$. 
Schittkowski, K. 2002. Numerical Data Fitting in Dynamical Systems-A Practical Introduction with Applications and Software. Kluwer Academic Publishers.

Schittkowski, K., 2007. Experimental design tools for ordinary and algebraic differential equations. Industrial \& Engineering Chemistry Research 46, 9137-9147.

Splechtna, B., Nguyen, T.H., Steinbo, C.K.M., Kulbe, K.D., Lorenz, W., Haltrich, D., 2006. Production of prebiotic galactooligosaccharides from lactose using $\beta$-galactosidase from Lactobacillus reuteri. Journal of Agricultural and Food Chemistry 54, 4999-5006.

Tuohy, K.M., Ziemer, C.J., Klinder, A., Knöbel, Y., Pool-Zobel, B.L., Gibson, G.R., 2002. A human volunteer study to determine the prebiotic effects of lactulose powder on human colonic microbiota. Microbial Ecology in Health and Disease 14, 165-173.

Walter, E., Pronzato, L., 1997. Identification of Parametric Models from Experimental Data. Springer. 